\title{
Thyroid Cancer Prevalence and Risk in Incidental Thyroid Lesions Detected with 18F-FDG PET/CT
}

\author{
(D) İbrahim Fatih Ceran'1, (D) Filiz Özülker², (D) Sevda Sağlampınar Karyağar22, (D) Tamer Özülker² \\ 1 University of Health Sciences Turkey, Yedikule Chest Diseases and Thoracic Surgery Training and Research Hospital, Clinic of Nuclear Medicine, \\ istanbul, Turkey \\ 2University of Health Sciences Turkey, Prof. Dr. Cemil Taşçıŏlu City Hospital, Clinic of Nuclear Medicine, İstanbul, Turkey
}

\section{Abstract}

Objective: The aim of this study is to analyze the relationship between thyroid nodules detected in patients who were examined in our clinic for any indication and had 18fluoro-fluorodeoxyglucose (18F-FDG) positron emission tomography/computed tomography (PET/CT) scanning and thyroid cancer.

Methods: Results of patients who had FDG PET/CT scanning for any indication other than thyroid cancer during 2015 and 2016 in 0 kmeydani Training and Research Hospital were examined. Age and genders of patients, FDG PET scanning indications, FDG accumulation type (focal or diffuse), lesion maximum standard uptake value $\left(S U V_{\max }\right)$, histology, and pathology of the patient who had fine-needle aspiration biopsy (FNAB) or surgery were recorded. According to the results obtained from these values, the relationship between thyroid nodules and thyroid cancer was analyzed.

Results: Among the group of 50 patients, 18F-FDG PET/CT scanning detected all as having thyroid nodules. Forty cases had benign diagnoses, whereas ten cases had malignant diagnoses either by FNAB or total thyroidectomy. According to the diagnosis results, the SUV max $_{\text {average }}$ of the malignant group was higher than the SUV $\mathrm{max}_{\text {ax }}$ average of the benign group. However, no statistically significant result was obtained $(p>0.05)$. The results also showed no statistically significant association regarding tumor size between the malignant and benign groups $(p>0.05)$.

Conclusion: Thyroid incidentaloma was detected among patients who had 18F-FDG PET/CT scanning for any reason other than thyroid cancer. Thyroid malignancy was detected in $20 \%$ of patients diagnosed with thyroid nodules by with focal $18 \mathrm{~F}$-FDG accumulation. No statistically significant results concerning SUV $V_{\max }$ value and lesion size were obtained among groups having benign and malignant diagnoses. Since no significant results were obtained between the SUV $V_{\max }$ and malignancy, no threshold value for SUV $V_{\max }$ was calculated.

Keywords: Thyroid nodules, incidentaloma, FDG PET/CT

\section{INTRODUCTION}

A nodule detected in the thyroid gland in imaging studies performed for different reason without PURPOSE imaging (1). 18Fluoro-fluorodeoxyglucose (18F-FDG) positron emission tomography (PET) is a valuable imaging method used for diagnosis, staging, restaging, treatment response, and biopsy guidance in oncological diseases (2).
A normal thyroid gland does not exhibit any physiological 18F-FDG uptake. However, in approximately $1.2 \%$ to $4.3 \%$ of the 18F-FDG PET studies, diffuse, or focal 18F-FDG uptake is observed incidentally (1-3). In this examination, $14 \%$ to $50 \%$ of cases with focal involvement have thyroid malignancy, and those with diffuse involvement have diffuse hyperthyroidism or thyroiditis (1-3). In a review article, the malignancy rate was $4.4 \%$ for diffuse involvement and $34.8 \%$ for focal involvement (4). Most 
studies conducted so far on this subject are retrospective and devoid of definite diagnostic algorithms. In the retrospective studies of incidental thyroid involvement, the final diagnosis has been reached in only $30 \%$ to $50 \%$ of cases (5-7). There are considerable limitations in systematic reviews on this subject, such as publishing bias and significant heterogeneity in studies (8).

We conducted this retrospective study to detect the rate of incidental involvement in the thyroid gland in 18F-FDG PET/ computed tomography (CT) examination in our clinic and to determine the rate of malignancy among these patients.

\section{METHODS}

\section{Patients}

In our study, 9,974 patients who underwent 18F-FDG PET/ CT imaging between January 2015 and December 2016 with indications other than thyroid diseases in the Nuclear Medicine Department of Okmeydani Training and Research Hospital were evaluated, and 50 patients with definitive histopathological diagnosis of incidental thyroid nodules were included in the study. Patients showing focal or diffuse FDG uptake in the thyroid gland in the 18F-FDG PET/CT study were evaluated and recorded as incidental involvement. Age, gender, 18F-FDG PET/CT imaging indication, 18F-FDG involvement (focal or diffuse), lesions' maximum standard uptake value $\left(S U V_{\text {max }}\right)$, and histopathology of those who underwent surgery or fine-needle aspiration biopsy (FNAB) were recorded. The histopathological diagnoses of patients who underwent FNAB were categorized as malignant, benign, and suspicious. Histopathological diagnoses of patients who underwent thyroidectomy and FNAB were examined and compared with 18F-FDG PET/CT findings. The cases with FNAB results that were inadequate, uncertain, and suspicious for malignancy (unless confirmed by total thyroidectomy) were excluded from the study. The incidence of incidental thyroid lesions, malignancy potentials of these lesions, the correlation of SUV $V_{\max }$ and $18 \mathrm{~F}-\mathrm{FDG}$ uptake patterns of lesions, with histopathological results of patients who underwent 18 F-FDG PET/ CT study were examined. A retrospective evaluation was made by obtaining 18 F-FDG PET/CT imaging results from the archive in the nuclear medicine department and histopathological results from the patient files and pathology archive. This retrospective study was conducted with the approval of the Medical Ethics Committee of Okmeydani Training and Research Hospital, with the decision dated March 14, 2018, and numbered 615. Informed consent was obtained from all individual participants included in the study.

\section{Imaging}

PET/CT imaging of patients included in the study was done with a Siemens Biograph 6 LSO HI-RES integrated PET/CT device (Siemens Medical Solutions, Knoxville, TN, USA). After four to six hours of fasting, patients waited in a quiet and dark room just before their exam. Blood glucose was measured with a glucometer just before the radiopharmaceutical injection. All patients used an oral contrast agent before imaging. Patients with blood glucose levels below $150 \mathrm{mg} / \mathrm{dL}$ were injected intravenously with 0.15 $0.20 \mathrm{mCi} / \mathrm{kg} 18 \mathrm{~F}-\mathrm{FDG}$. After a 60 -minute waiting period, the patient's bladder was emptied, and the patient was placed in the supine position on the bed of the PET/CT scanner. First guideline topogram CT images were acquired, then subsequent PET images of body parts from the vertex to the proximal thigh were acquired. The images of the patients were completed in approximately 20 to 25 minutes, with an average of 7 to 8 bed positions. The CT portion of the PET/CT study was non-diagnostic and used for attenuation correction and anatomical localization of 18F-FDG PET images.

\section{Histopathological Evaluation}

The biological material obtained while performing the diagnostic and treatment procedures (FNAB or thyroidectomy) was examined histopathologically in our hospital's pathology department, and immunohistopathological staining was performed as necessary.

\section{Statistical Analysis}

IBM SPSS Statistics 22 for statistical analysis (SPSS IBM, Turkey) program was used to evaluate the findings of the study. The appropriateness of the parameters to normal distribution was evaluated with the Shapiro-Wilk test. In addition to descriptive statistical methods (mean, standard deviation, frequency), in the comparison of quantitative data, Student's t-test was used for comparing the parameters that showed normal distribution, and the Mann-Whitney $\mathrm{U}$ test was used for parameters that did not show normal distribution. Fisher's exact test was used to compare qualitative data. Significance was evaluated at the level of $p<0.05$.

\section{RESULTS}

Incidental involvement in the thyroid gland was observed in 401 (4\%) of patients who underwent 18F-FDG PET/CT (focal $2.3 \%$, diffuse 1.6\%). Diffuse involvement was observed in 162 (41\%) (Figure 1) and focal uptake in 239 (59\%) of these patients. Histopathological diagnosis was obtained by FNAB in 43 patients, and surgery in 7 patients. The average patient 
age was $60.84 \pm 13.01$ (34-86) years. Ten (20\%) were male, and $40(80 \%)$ were female. Our study parameters included were $\mathrm{SUV}_{\max }$, histopathological diagnosis and incidental lesion tumor size (Table 1). SUV $\max$ range from 2.73 to 29.27 with an average of $8.39 \pm 5.83$. The average $S \mathrm{SV}_{\max }$ for benign and malignant lesions were $7.56 \pm 3.81$ (mean, 6.15) and 11.72 \pm 10.34 (mean, 7.18).

Tumor sizes ranged from 6 to $43 \mathrm{~mm}$, and the average size was $19.47 \pm 9.27 \mathrm{~mm}$. The size of the nodules, either malignant or benign, was $\leq 15 \mathrm{~mm}$ in 23 cases (46\%) and $\geq 15 \mathrm{~mm}$ in 27 cases (54\%). While $40(80 \%)$ of 50 cases were diagnosed as benign, (Figure 2) 10 (20\%) were diagnosed as malignant (Figure 3). When the size assessment was made in malignant cases, 6 of 10 were $15 \mathrm{~mm}$ and below. Although the malignancy rate was higher in nodules smaller than $15 \mathrm{~mm}$, it was statistically insignificant. In benign nodules, 17 of 40 cases had a size of 15 $\mathrm{mm}$ or less.

Six out of 23 nodules (26\%) smaller than $15 \mathrm{~mm}$ were found to be malignant. This finding was higher than the malignancy rate in all 18 F-FDG positive nodules (20\%) but was not statistically significant. The average SUV max $_{\text {mere }}$ higher in the malignant group, but there was no statistically significant difference regarding $S_{U V} V_{\max }(p>0.05)$. Since there was no statistically significant difference between the $\mathrm{SUV}_{\max }$ averages of

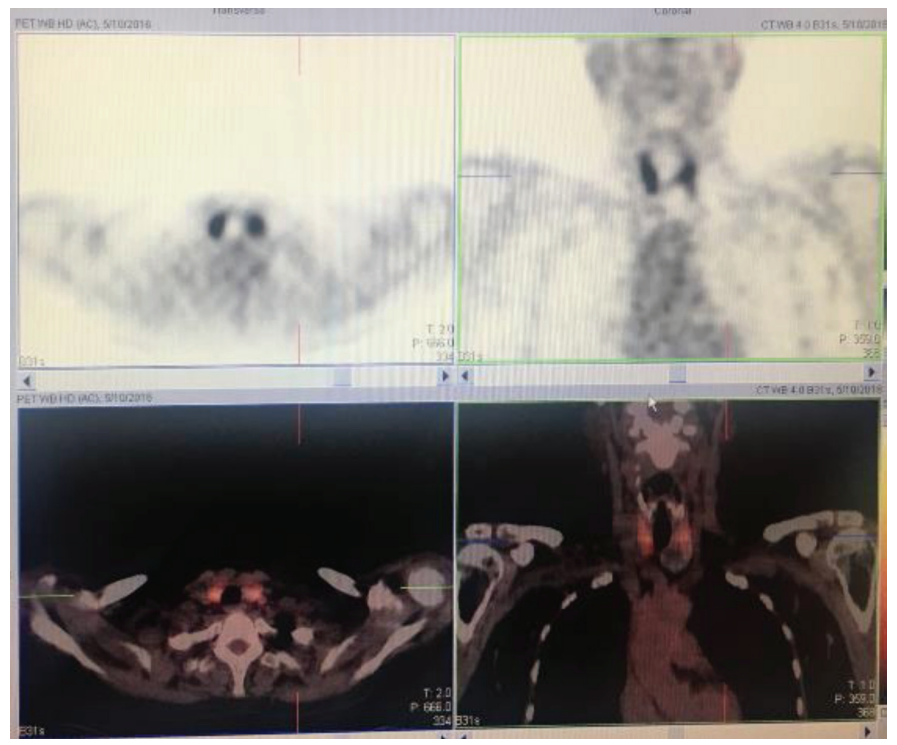

Figure 1. Chronic thyroiditis pattern showing diffuse FDG uptake. A 56-year-old female patient underwent $18 \mathrm{~F}$-FDG PET/CT for restaging of cervical cancer. Axial PET and fusion images (left column) and coronal PET and fusion images (right column) show incidental diffuse FDG uptake in the thyroid gland (SUV $\max _{\text {: }}$ 14.69). The patient underwent FNAB, and her histopathology revealed lymphocytic thyroiditis

${ }^{18} \mathrm{~F}-\mathrm{FDG}$ : ${ }^{18}$ Fluoro-fluorodeoxyglucose, PET: Positron emission tomography, CT:

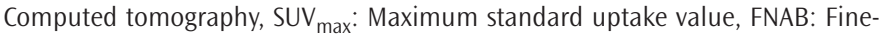
needle aspiration biopsy malignant and benign nodules, a cut-off point could not be calculated.

\section{DISCUSSION}

Incidence of detecting thyroid nodules on the neck ultrasonography (USG) performed for a purpose other than the thyroid gland is between $14 \%$ and $46 \%$ (1). The nodule detection rate varies among different radiological imaging methods.

\begin{tabular}{|c|c|c|c|}
\hline & \multicolumn{2}{|l|}{ Diagnosis } & \multirow[t]{2}{*}{$\mathrm{p}$} \\
\hline & Benign & Malignant & \\
\hline SUV $_{\max }$ Avg. \pm SD (median) & $\begin{array}{l}7.56 \pm 3.81 \\
(6.15) \\
\end{array}$ & $\begin{array}{l}11.72 \pm 10.34 \\
(7.18)\end{array}$ & 10.689 \\
\hline \multicolumn{4}{|l|}{ Tumor size (n, \%) } \\
\hline$\leq 15 \mathrm{~mm}$ & $17(42.5 \%)$ & $6(60 \%)$ & \multirow{2}{*}{20.480} \\
\hline$>15 \mathrm{~mm}$ & $23(57.5 \%)$ & $4(40 \%)$ & \\
\hline Age mean $\pm S D$ & $61.9 \pm 12.59$ & $56.6 \pm 14.49$ & 30.253 \\
\hline \multicolumn{4}{|l|}{$\operatorname{Sex}(n, \%)$} \\
\hline Male & $8(20 \%)$ & $2(20 \%)$ & 21.000 \\
\hline Female & $32(80 \%)$ & $8(80 \%)$ & \\
\hline
\end{tabular}

${ }^{1}$ Mann-Whitney $U$ test, ${ }^{2}$ Fisher's exact test, ${ }^{3}$ Student's t-test, SUV $_{\text {max }}$ : Maximum standard uptake value, Avg.: Average, SD: Standard deviation

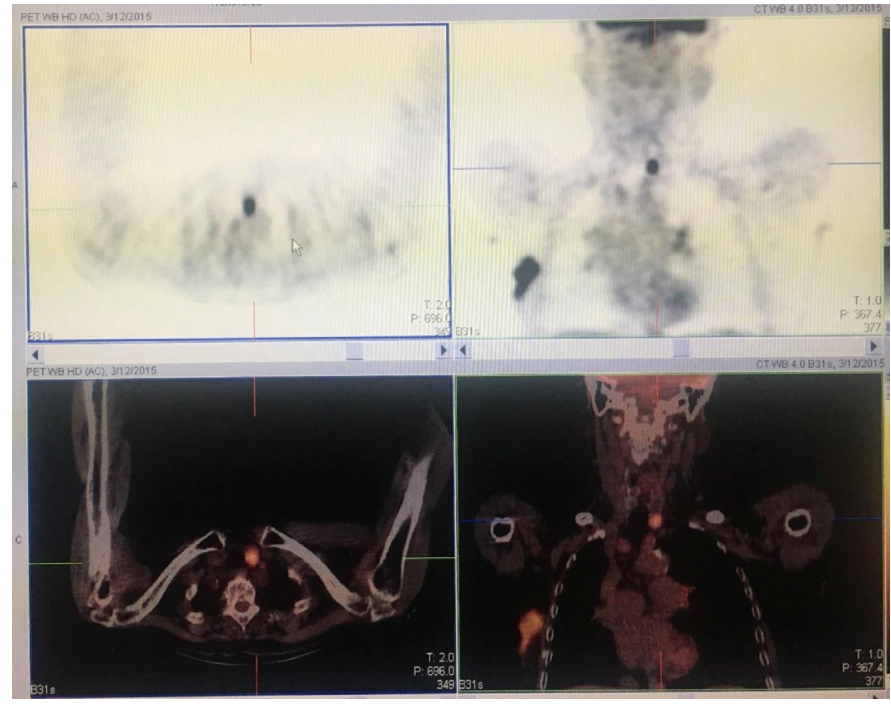

Figure 2. Benign thyroid nodule that shows focal FDG uptake. An 86-year-old female patient underwent an 18F-FDG PET/CT study for the evaluation of cancer of unknown primary origin. Axial PET and fusion images (left column) and coronal PET and fusion images (right column) show focal FDG uptake in the left lobe of the thyroid gland. (SUV $\max$ : 12.91). Correlation with USG showed a $15 \mathrm{~mm}$ nodule, and the FNAB results from this nodule were benign

${ }^{18} \mathrm{~F}-\mathrm{FDG}$ : ${ }^{18}$ Fluoro-fluorodeoxyglucose, PET: Positron emission tomography, CT: Computed tomography, SUV $\mathrm{V}_{\max }$ : Maximum standard uptake value, FNAB: Fineneedle aspiration biopsy, USG: Ultrasonography 
Thyroid gland incidentalomas are seen in 16\% of CT and magnetic resonance imaging studies and 27\% of neck ultrasonographies $(9,10)$. In an autopsy series, incidental nodules were more common in the thyroid gland. Mortensen et al. (11) reported that macroscopically at least one nodule was observed in half of the patients, and nodules were larger than $2 \mathrm{~cm}$ in approximately $33 \%$ of all cases.

The incidental detection rate of $18 \mathrm{~F}-\mathrm{FDG}$ avid thyroid nodules in $18 \mathrm{~F}$-FDG PET/CT studies has been reported between $0.1 \%$ and $4.3 \%$ (12). In our retrospective study, incidental uptake in the thyroid gland, either focal or diffuse, was observed in 401 (4\%) of 9,974 patients who underwent 18F-FDG PET/CT for evaluation of different malignancies. It is known that thyroid nodules are more common in women than in men. Also, 18F-FDG PET/CT incidentalomas are more common in women (1). In our cohort, $74 \%$ of incidentalomas were detected in women. The nodule observed in the focal involvement area located incidentally in the thyroid gland is likely to be malignant. Therefore, the presence of nodules that show focal 18 F-FDG uptake should be evaluated with USG, and FNAB should be performed if necessary (13).

In our study, 60 of 239 patients with focal involvement underwent FNAB, and seven of them had a total

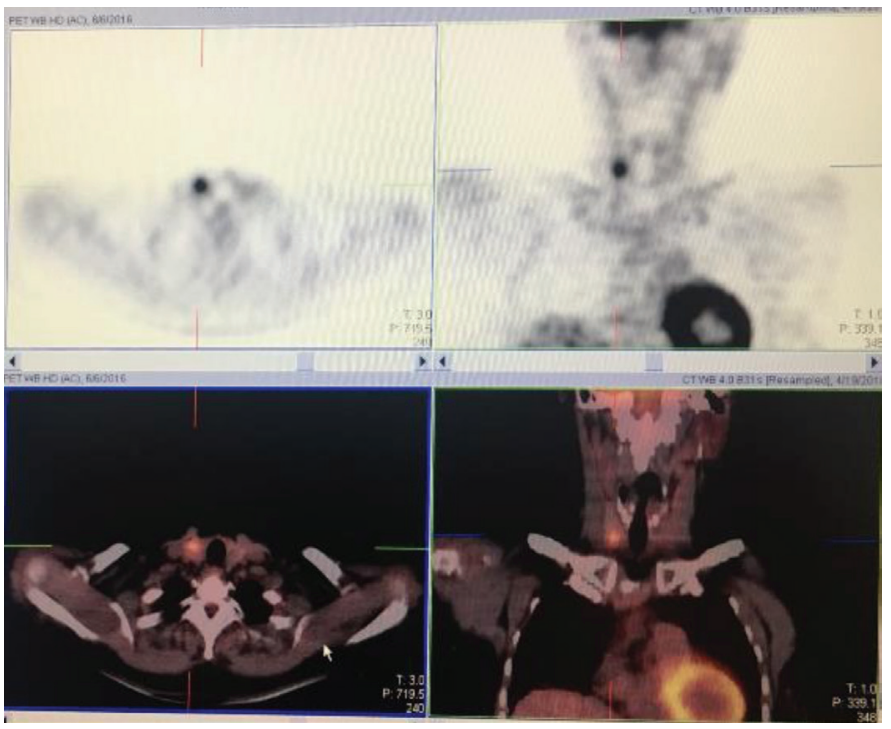

Figure 3. Malignant thyroid nodule that shows focal FDG uptake. A 46-year-old female patient underwent an $18 \mathrm{~F}$-FDG PET/CT study for the evaluation of cancer of unknown primary origin. Axial PET and fusion images (left column) and coronal PET and fusion images (right column) show focal FDG uptake in the right lobe of the thyroid gland. (SUV $\max$ : 7.8). Ultrasonography showed an $11 \mathrm{~mm}$ nodule corresponding to the focal uptake. FNAB from the nodule revealed atypia of unknown significance. Subsequently, the patient underwent a total thyroidectomy, and the pathology results revealed papillary thyroid carcinoma

${ }^{18} \mathrm{~F}-\mathrm{FDG}$ : ${ }^{18}$ Fluoro-fluorodeoxyglucose, PET: Positron emission tomography, CT:

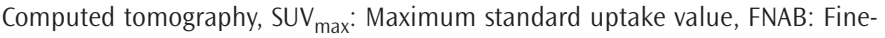
needle aspiration biopsy thyroidectomy in addition to FNAB. Malignancy was detected in ten of the patients with malignancy-compatible cytology after total thyroidectomy and/or FNAB and was used as the gold standard to diagnose thyroid cancer. The risk of malignancy was calculated as 20\% (10/50) for patients without FNAB. Inadequate and uncertain FNAB results and suspected malignant cases of FNAB (unless confirmed by total thyroidectomy) were excluded from the study. In a systematic review on this subject, the malignancy rates obtained with total thyroidectomy and FNAB in patients with incidental focal $18 \mathrm{~F}$-FDG involvement in the thyroid gland were reported as $29.3 \%-33.2 \%$ (8). Bae et al. (7) reported the malignancy rate as $30.9 \%$ in patients with focal $18 \mathrm{~F}$-FDG involvement in the thyroid gland. These rates are somewhat higher than the rates we found (20\%). The probable reason for obtaining a rate below those cited in the literature is the low number of patients who had FNAB or total thyroidectomy, which is also a limitation of the study. However, cancer rates of focal incidentalomas were higher and were from $41.7 \%$ to $47 \%$ in some studies. However, these studies were limited and included a low number of patients in the study and made the histopathological diagnosis mostly by FNAB instead of an operation $(14,15)$. Chen et al. (16) reported the malignancy rate of the $18 \mathrm{~F}-\mathrm{FDG}$ positive thyroid nodules as $14 \%$, a rate lower than that found in the literature. We think that the low risk of malignancy in this study may be due to bias in the evaluation of FNAB results on focal incidentalomas. In addition, geographical differences in which the study was conducted may be cause discordant malignancy risk.

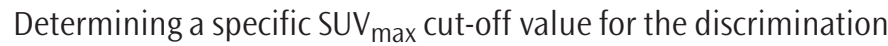
of benign and malignant thyroid nodules is another concern. Makis and Ciarallo (12) reported a statisticallysignificant difference between the median $S U V_{\max }$ of benign thyroid incidentalomas (SUV $\left.V_{\max }: 4.8\right)$ vs malignant (SUV max $_{\text {: }}$ 6.3). However, due to the wide range of overlap between the two groups, the authors did not find it reliable to determine the malignancy with this SUV $\max$ threshold value. Pagano et al. (17) stated that malignant lesions have a higher SUV $V_{\max }$ than benign lesions, and this has a positive correlation when SUV $\max :>5$. The meta-analyses also reported a significant difference between SUV max $_{\max }$ of benign lesions and malignant lesions. However, no particular SUV $V_{\max }$ cut-off value was identified because of the overlap between the two groups $(4,8,18)$. In our study, the average SUV $_{\max }$ was higher in the group diagnosed as malignant. Still, the difference between benign and malignant nodules regarding SUV $V_{\max }$ averages was not statistically significant $(p>0.05)$. 
In a study conducted by Shi et al. (19) with 5,216 patients, the malignant thyroid incidentalomas were significantly larger than benign lesions (malignant $1.8 \pm 0.8 \mathrm{~cm}$, benign $1.3 \pm 0.5 \mathrm{~cm}$, $p=0.006)$. They had higher $\operatorname{SUV}_{\max }(11.3$ vs. $4.8, p<0.001)$. We chose a $1.5 \mathrm{~cm}$ cut-off value arbitrarily. There was no statistically significant difference regarding the distribution rates between the tumor size groups $(p>0.05)$.

The 2015 American Thyroid Association (ATA) guideline suggests that if the uptake is diffuse, it is confirmed by USG or clinical findings regarding the $18 \mathrm{~F}-\mathrm{FDG}$ involvement in the thyroid gland. There is no need for further imaging examination (strong recommendation medium level of evidence). In focal 18 F-FDG uptake, if the nodule is confirmed with USG and the size of the nodule is $>1 \mathrm{~cm}$, thyroid FNAB is recommended because the risk of malignancy increases (strong advice medium evidence) (20). Similarly, the 2016 Agency for Care Effectiveness (ACE) guideline recommends FNAB for increased risk of malignancy in thyroid nodules that show focal 18F-FDG uptake on PET. However, if the involvement is diffuse, they do not recommend further investigation. Unlike the ATA guideline, the ACE guideline does not make any recommendations regarding the nodule size for performing a biopsy of the nodule that shows 18F-FDG uptake (21).

\section{CONCLUSION}

Thyroid incidentaloma was detected in $4 \%$ of patients who underwent 18F-FDG PET/CT for non-thyroid cancer. The pattern of FDG uptake in these patients was focal in 59\% and diffuse in $41 \%$. Thyroid malignancy was detected in $20 \%$ of patients with 18F-FDG PET/CT with focal FDG uptake. There was no significant difference between the groups diagnosed benign and malignant concerning SUV $V_{\max }$ and lesion size. The cut-off value for $S U V_{\max }$ could not be calculated because there was no relationship between SUV $V_{\max }$ and malignancy.

It was thought that thyroid incidentalomas that show focal 18F-FDG uptake on 18F-FDG PET/CT studies should be correlated with USG. Because of the high risk of malignancy, analysis of histopathologies should be performed with FNAB.

\section{Ethics}

Ethics Committee Approval: This retrospective study was conducted with the approval of the Medical Ethics Committee of Okmeydani Training and Research Hospital, with the decision dated March 14, 2018, and numbered 615.
Informed Consent: Informed consent was obtained from all individual participants included in the study.

Peer-review: Externally and internally peer-reviewed.

\section{Authorship Contributions}

Surgical and Medical Practices: I.F.C., Concept: I.F.C., T.Ö., Design: I.F.C., T.Ö., Data Collection or Processing: I.F.C., Analysis or Interpretation: I.F.C., T.Ö., Literature Search: I.F.C., Writing: I.F.C., F.Ö., S.S.K.

Conflict of Interest: No conflict of interest was declared by the authors.

Financial Disclosure: The authors declared that this study received no financial support.

\section{REFERENCES}

1. Jin J, Wilhelm SM, McHenry CR. Incidental thyroid nodule: patterns of diagnosis and rate of malignancy. Am J Surg 2009;197:320-4.

2. Yasuda S, Ide M, Fujii H, Nakahara T, Mochizuki Y, Takahashi W, et al. Application of positron emission tomography imaging to cancer screening. Br J Cancer 2000;83:1607-11.

3. Choi JY, Lee KS, Kim HJ, Shim YM, Kwon OJ, Park K, et al. Focal thyroid lesions incidentally identified by integrated 18F-FDG PET/CT: clinical significance and improved characterization. J Nucl Med 2006;47:60915.

4. Soelberg KK, Bonnema SJ, Brix TH, Hegedüs L. Risk of malignancy in thyroid incidentalomas detected by $18 \mathrm{~F}$-fluorodeoxyglucose positron emission tomography: a systematic review. Thyroid 2012;22:918-25.

5. Kwak JY, Kim EK, Yun M, Cho A, Kim MJ, Son EJ, et al. Thyroid incidentalomas identified by 18 F-FDG PET: sonographic correlation. AJR Am J Roentgenol 2008;191:598-603.

6. Chen W, Parsons M, Torigian DA, Zhuang H, Alavi A. Evaluation of thyroid FDG uptake incidentally identified on FDG-PET/CT imaging. Nucl Med Commun 2009;30:240-4.

7. Bae JS, Chae BJ, Park WC, Kim JS, Kim SH, Jung SS, et al. Incidental thyroid lesions detected by FDG-PET/CT: prevalence and risk of thyroid cancer. World J Surg Oncol 2009;7:63.

8. Shie P, Cardarelli R, Sprawls K, Fulda KG, Taur A. Systematic review: prevalence of malignant incidental thyroid nodules identified on fluorine-18 fluorodeoxyglucose positron emission tomography. Nucl Med Commun 2009;30:742-8.

9. Brander A, Viikinkoski P, Nickels J, Kivisaari L. Thyroid gland: US screening in a random adult population. Radiology 1991;181:683-7.

10. Youserm DM, Huang T, Loevner LA, Langlotz CP. Clinical and economic impact of incidental thyroid lesions found with CT and MR. AJNR Am J Neuroradiol 1997;18:1423-8.

11. Mortensen JD, Woolner LB, Bennett WA. Gross and microscopic findings in clinically normal thyroid glands. J Clin Endocrinol Metab 1955; 15:1270-80

12. Makis W, Ciarallo A. Thyroid Incidentalomas on 18F-FDG PET/CT: Clinical Significance and Controversies. Mol Imaging Radionucl Ther 2017;26:93-100. 
13. American Thyroid Association (ATA) Guidelines Taskforce on Thyroid Nodules and Differentiated Thyroid Cancer, Cooper DS, Doherty GM, Haugen BR, Kloos RT, Lee SL, et al. Revised American Thyroid Association management guidelines for patients with thyroid nodules and differentiated thyroid cancer. Thyroid 2009;19:1167-214.

14. Nam SY, Roh JL, Kim JS, Lee JH, Choi SH, Kim SY. Focal uptake of (18) F-fluorodeoxyglucose by thyroid in patients with nonthyroidal head and neck cancers. Clin Endocrinol (Oxf) 2007;67:135-9.

15. Cohen MS, Arslan N, Dehdashti F, Doherty GM, Lairmore TC, Brunt LM, et al. Risk of malignancy in thyroid incidentalomas identified by fluorodeoxyglucose-positron emission tomography. Surgery 2001;130:941-6.

16. Chen W, Parsons M, Torigian DA, Zhuang H, Alavi A. Evaluation of thyroid FDG uptake incidentally identified on FDG-PET/CT imaging. Nucl Med Commun 2009;30:240-4.

17. Pagano L, Samà MT, Morani F, Prodam F, Rudoni M, Boldorini R, et al. Thyroid incidentaloma identified by $18 \mathrm{~F}$-fluorodeoxyglucose positron emission tomography with CT (FDG-PET/CT): clinical and pathological relevance. Clin Endocrinol (Oxf) 2011;75:528-34.
18. Bertagna F, Treglia G, Piccardo A, Giubbini R. Diagnostic and clinical significance of F-18-FDG-PET/CT thyroid incidentalomas. J Clin Endocrinol Metab 2012;97:3866-75.

19. Shi H, Yuan Z, Yuan Z, Yang C, Zhang J, Shou Y, et al. Diagnostic Value of Volume-Based Fluorine-18-Fluorodeoxyglucose PET/CT Parameters for Characterizing Thyroid Incidentaloma. Korean J Radiol 2018;19:342-51.

20. Haugen BR, Alexander EK, Bible KC, Doherty GM, Mandel SJ, Nikiforov YE, et al. 2015 American Thyroid Association Management Guidelines for Adult Patients with Thyroid Nodules and Differentiated Thyroid Cancer: The American Thyroid Association Guidelines Task Force on Thyroid Nodules and Differentiated Thyroid Cancer. Thyroid 2016;26:1133.

21. Gharib H, Papini E, Garber JR, Duick DS, Harrell RM, Hegedüs L, et al. American association of clınıcal endocrınologists, amerıcan college of endocrınology, and associazıone medicı endocrınologı medıcal guıdelınes for clınıcal practıce for the diagnosıs and management of thyroıd nodules--2016 UPDATE. Endocr Pract 2016;22:622-39. 